\title{
EDITORIALE
}

\section{Il disagio della medicina}

\author{
The malaise of medicine
}

La medicina vive un profondo disagio. Lo vive su molti piani: quello del suo sapere, della sua prassi, della sua organizzazione, dei suoi rapporti con i cittadini. Lo testimonia un senso diffuso di scontento che pervade i medici e gli infermieri, così come i pazienti e quanti lavorano nella sanità sotto il profilo manageriale. Ne sono espressione i numerosi articoli che vengono pubblicati nelle riviste mediche in cui questo disagio viene fondamentalmente ricondotto a quella che viene indicata come "medicina della complessità".

Non vi è dubbio che la tipologia attuale dei nostri pazienti sia maggiormente carica di problemi rispetto a quanto si osservava alcuni decenni fa. L'invecchiamento della popolazione, infatti, ha portato con sé un notevole aumento della comorbosità, cui hanno contribuito anche la maggiore disponibilità ed efficacia dei mezzi d'indagine e il vasto impiego di farmaci attivi ma gravati da problemi di interazione e di effetti collaterali. Soprattutto, va tenuto conto del fatto che le condizioni socioeconomiche, culturali, comportamentali, ambientali contribuiscono esse pure alla complessità, senza che questi aspetti, in tutte le loro ramificazioni, abbiano ricevuto un'adeguata attenzione [1]. Insomma, bisogna considerare che il paziente si presenta a noi con le sue caratteristiche somatiche e psichiche in cui entrano in gioco gli aspetti biologico-genetici che gli sono propri, ma in cui rientrano anche, e potentemente, il suo inserimento sociale, il limite delle disponibilità economiche personali per curarsi, il livello culturale, l'ambiente di vita, la maggiore o minore distanza dai "centri di salute", la capacità o l'incapacità di muoversi autonomamente e di avere autonomamente cura di sé. Tutto questo non può essere trascurato, anche se gli studi in cui questi aspetti siano stati presi in considerazione - non certo facili da condurre - sono scarsi e limitati soltanto ad alcuni aspetti. Ciò nonostante, la loro necessità assume particolare rilevanza in una società multietnica e multiculturale come ormai è anche la nostra.

Un altro motivo di disagio è costituito dalla cronicità: i nostri malati sono sempre più spesso afflitti da patologie che riusciamo a controllare, ma molto meno spesso a guarire. Il nostro modello medico - pensiamo soprattutto agli ospedali
- prevedeva invece una sanità di guariti o di morti e assai meno di cronici, per i quali ultimi vi erano le "lunghe degenze", già molti anni fa piuttosto carenti. Sarebbe pertanto necessario «ridisegnare il sistema della salute per adeguarlo all'accresciuto peso delle malattie croniche in una popolazione di anziani» [2]. Una "medicina della cronicità" - se così possiamo esprimerci - richiederebbe di considerare nel paziente non soltanto la sua o le sue malattie (diseases), ma anche il suo modo di vivere e di sperimentare questa o queste malattie, cioè la sua illness; richiederebbe uno stretto e positivo rapporto tra paziente e medico di famiglia; richiederebbe, infine, un adattamento dell'organizzazione politica, sociale ed economica a queste nuove esigenze che costituiscono un ulteriore aspetto della medicina della complessità [2].

In sostanza, sono cambiati i malati con cui il medico d'oggi deve confrontarsi: cambiati non soltanto per i mutamenti del quadro patologico che l'invecchiamento della popolazione e le capacità di soppressione dei sintomi di molti farmaci hanno comportato, ma anche perché è mutato il quadro socioeconomico della popolazione. E come se tutto ciò non bastasse, è mutato anche il quadro filosofico in cui la medicina, in quanto scienza, si pone di fronte ai suoi problemi. Scrive Atar Yawar in un recente editoriale su Lancet: «La verità nel senso letterale del termine è divenuta obsoleta circa cento anni fa nella scienza e nell'arte. I fisici hanno riscoperto che il modo in cui la realtà ci appare dipende dalle condizioni dell'osservatore; la materia è stata vista come un'espressione dell'energia. L'arte è divenuta cubista, dadaista, surrealista e postmoderna. La filosofia postmoderna nasce dalla considerazione che il racconto modernista di un progresso materiale senza fine ha causato vittime non riconosciute e intrinseche assurdità in cui gli esseri umani possono sprofondare» [3].

La consapevolezza dell'impossibilità pratica di istituire il trattamento ideale per tutti malati e per tutte le condizioni considerate patologiche accresce il nostro senso di sgomento. In un commento all'editoriale di Yawar sopra citato si è affermato che la scienza della complessità rappresenta il 
neoippocratismo del XXI secolo, che dovrebbe tenere insieme arte e scienza per costruire un sapere olistico capace di accettare i caratteri dell'incertezza, dell'irregolarità e della soggettività, più confacenti a ciò che la medicina, oggi, può e deve essere [4].

Ciò che possiamo affermare è che la consapevolezza della nuova complessità della medicina si è ormai fatta strada tra $i$ medici. È necessario, tuttavia, che questa consapevolezza riesca a evolvere in un mutamento di paradigma nell'accezione kuhniana del termine - per Kuhn il termine paradigma indica le coordinate che in un dato periodo orientano la scienza («scienza normale») sulla base di acquisizioni «sufficientemente nuove» e «sufficientemente aperte» [5] - affinché l'attuale disagio possa trovare una composizione in una medicina rinnovata in cui scienza e arte collaborino a interpretare le malattie more scientifico e a comprendere il malato more humano, come già molti anni fa reclamava Enrico Poli [6].

Un'inchiesta che ricostruisce la figura del medico nel Friuli tra gli anni Cinquanta e Ottanta del secolo appena trascorso riporta: «Un uomo serio, si può dire anche brusco, però severo e bravo, era un medico. Ha salvato molta gente, anche bambini, gente con la pleurite, li ha curati a casa. Quella volta non si mandava in ospedale», «Medico, levatrice e farmacista non avevano orari di chiusura, giorno e notte sempre aperti. Una volta si era più assistiti, c'erano meno dottori e più assistenza, sia a casa che in ospedale» [7].

Non pensiamo a inutili e impossibili ritorni al passato. $\mathrm{Ci}$ pare soltanto che se condizioni, modi e mezzi di allora non siano da recuperare, lo debbano essere invece quello spirito di servizio, quella disponibilità e quell'approccio umano senza i quali la medicina resta soltanto una "tecnica riparativa". Spetta però alla classe politica e amministrativa l'onere di creare le condizioni per questa medicina al tempo stesso nuova e antica. Una medicina maggiormente capace di soddisfare $i$ pazienti e meno costosa di quella che crede di risolvere tutto richiedendo acriticamente quanto la tecnologia offre di più costoso. Vorremmo che qualcuno potesse tornare a dire di noi, soltanto e semplicemente, "è un medico".

\section{Bibliografia}

[1] Safford MM, Allison JJ, Kiefe Cl. Patient complexity: more than comorbidity. The vector model of complexity. J Gen Intern Med 2007;22 Suppl 3:382-90.

[2] Martin C, Sturmberg J. Complex adaptive chronic care. J Eval Clin Pract 2008;15:571-7.

[3] Yawar A. The art of medicine: medicine and the human story. Lancet 2010;375:546-7.

[4] Rambihar VS, Rambihar VS. Complexity: the science for medicine and the human story. Lancet 2010;375:1162.

[5] Kuhn T. La struttura delle rivoluzioni scientifiche. Tr it. Torino: Einaudi; 1999. p. 29-30.

[6] Poli E. Metodologia medica: principi di logica e pratica clinica. Milano: Rizzoli; 1965. p. 19.

[7] Simeoni M. Un medico condotto in Italia, il passato e il presente: un'analisi qualitativa. Milano: Franco Angeli, 2009: p. 148,150.

$$
\begin{array}{r}
\text { Vito Cagli" } \\
\text { Socio Onorario FADOI Lazio } \\
\text { Emeritus Editor dell'Italian Journal of Medicine } \\
\text { "E-mail: v.cagli@alice.it }
\end{array}
$$

